\title{
MODERNIDAD Y ACTUALIDAD DE MONTAIGNE
}

\section{Joan Lluis Llinas Begon*}

Michel de Montaigne está de moda. Se le considera uno de los pensadores más representativos del siglo XVI, se le vindica como un autor importante para entender la modernidad y la crisis del pensamiento actual, pero, sin embargo, su presencia en las historias de la filosofía suele ser mínima, cuando no nula. Quizás sea debido a la poca importancia filosófica que habitualmente se concede al siglo XVI, siglo sin grandes pensadores sistemáticos, simple transición entre el orden medieval y el moderno. Quizás se deba también a la gradación que parece establecerse entre los términos pensador y filósofo por lo que hace a la profundidad y sistematicidad del pensamiento. Visto de esta manera Montaigne sería un pensador interesante, pero no poseería méritos suficientes para figurar entre los filósofos que merecen un capítulo en la historia de la filosofía. Ese evitar la denominación de filósofo se extiende a los propios montaigneanos, como se puede comprobar echando una ojeada a la numerosa bibliografía existente sobre Montaigne: pocos son los títulos que se atreven a usar los términos "filosofía" y "filósofo". De hecho, en apoyo de esa posición se puede presentar una afirmación del propio Montaigne: "No soy filósofo" (III,9,927d $)^{1}$. Y sin embargo conviene tener en cuenta que a lo largo del millar de páginas de los Ensayos este término, como muchos otros, es tilizado de modo diverso. Cierto es que en el libro aparece una visión negativa de la filosofía, y que Montaigne se desmarca de esa imagen, pero también lo es que se dibuja otra filosofía, la que forma el juicio y las costumbres, con cuya actividad se puede asociar la actividad de Montaigne como productor de los Ensayos. En lo que sigue sugeriré lo siguiente: 1. Que

\footnotetext{
* Universitat de les Illes Balears (UIB), Palma de Mayorca, España; jlluis.llinas@uib.es

1 "Je ne suis pas philosophe". La primera cifra remite al número de libro de los Ensayos, la segunda al capítulo, la tercera a la página de la edición de Thibaudet y Rat, Montaigne. Oeuvres complètes., Gallimard, Paris, 1962. La letra se refiere a la edición de los Ensayos: a para la edición de 1580, b para la de 1588, c para los añadidos posteriores a esa fecha.
} 
Montaigne no sólo es un filósofo, sino que mantiene una posición original sobre lo que significa la actividad filosófica; 2 . Que la lectura de Montaigne, teniendo en cuenta tanto lo que dice como lo que hace, puede ser útil para reflexionar sobre algunos temas planteados en esta época de crisis de la modernidad.

Veamos, en primer lugar, cuál es la filosofía que rechaza Montaigne y con la que no quiere ser asimilado. Esa filosofía la identifica Montaigne con la lógica y con la metafísica. La lógica es una disciplina que regula el pensamiento, $y$, por tanto, es útil. El problema aparece cuando se considera la lógica no como un instrumento sino como un fin en sí mismo, cuando se reduce la filosofía a lógica. La filosofía limitada a un mero ejercicio verbal, a un punto en que los nombres abandonan su significación concreta, pierde cualquier utilidad que pudiera tener. Por su parte, la metafísica es rechazada en la medida que se presenta como disciplina capaz de explicar el mundo, poseedora de la verdad y la certeza (II,12,556a). Muchas páginas del capítulo más famoso de los Ensayos, la "Apología de Raimundo Sabunde" (II,12), se dedican a dejar patente la vanidad de la filosofía que pretende conocer la verdad del mundo y que, en esa arrogante tarea, no consigue más que poner de manifiesto su ignorancia. Los argumentos que Montaigne aporta los recoge sobre todo de Sexto Empírico y de Agrippa, y puesto que son bien conocidos -han sido la causa principal que ha hecho que Montaigne ecibiera el calificativo de escéptico- y no son propiamente el objeto de este escrito, no serán recordados aquí2.

En cambio, Montaigne habla a menudo de otra filosofía, útil en tanto que permite formar el juicio y las costumbres. La imagen de los juegos olímpicos, atribuida a Pitágoras y recogida por Montaigne a través de Cicerón, puede servir para ilustrarla $(1,26,157 c)$. El mundo es un gran estadio. Allí encontramos los que entrenan su cuerpo para triunfar en el estadio y alcanzar la gloria, y los que llevan cosas para vender y extraer de

2 Estos argumentos están recogidos en la mayoría de los estudios sobre Montaigne. Por citar el más clásico: Pierre Villey, Les sources et l'évolution des Essais de Montaigne. Hachette, Paris, 1908, 2 vols. O, más específicamente, véase también otro clásico: Richard Popkin, The History of Scepticism from Erasmus to Spinoza, Berkeley, University of California Press. Hay traducción castellana de Juan José Utrilla, FCE, México, 1983. 
ellas un beneficio. Finalmente, los hay, y no son los peores, que van al estadio para mirar cómo y por qué se hace cada cosa, y para ser espectadores de la vida de los otros hombres, con el objetivo de regular la suya. Esa actitud de observación es la propia del filósofo, pero no se trata de una observación pura, de un simple espectador, sino de un espectador interesado, puesto que de la observación debe obtener respuesta a cómo vivir. La filosofía, pues, es básicamente filosofía moral.

Pero tampoco la filosofía moral es ajena a la crítica de Montaigne. Por una parte, le afecta una de las principales críticas a la metafísica, la imposibilidad de concluir definitivamente una verdad. La filosofía moral no puede ser sólo un conjunto de preceptos que se presenten como soluciones verdaderas. Por otra parte, la filosofía, para ser efectiva, no puede distanciarse del sujeto que la practica. Es una persona concreta la que observa en el estadio con el objetivo de regular su vida. El lenguaje, instrumento de la filosofía, se sitúa para Montaigne por debajo de la vivencia: nos dice, por ejemplo, que los discursos filosóficos sobre la amistad no pueden igualarse al hecho de vivir una auténtica amistad $(1,28,192 a)$. Ello es debido a que la palabra es igualadora, eliminadora de diferencias $y$, por tanto, falsificadora. La única manera que puedan ser útiles en nuestra formación es que sean asimilados por quien los produce o los capta. De hecho, tanto la crítica a la lógica como a la metafísica se basan en último término en rechazar un discurso que es sólo palabras, puro artificio, un discurso externo y extraño al sujeto. La filosofía moral a veces cae en esa trampa y de ahí que produzca discursos cuyo cumplimiento es imposible, proponiendo reglas sobrehumanas (II,12,657b; III,9,967b).

Si bien Montaigne entiende la filosofía como filosofía moral, que califica de "verdadera y natural filosofía" $(1,39,242 a)^{3}$, ésta no puede ser mero discurso, sino que tiene que ser un discurso que afecte a la conciencia (III,9,967-8b). No consiste en unos determinados contenidos sino en cómo se entiende esa actividad, en cómo se produce en el sujeto la asimilación del discurso, en cómo éste se impregna en el alma, se diluye en el sujeto y deja de ser un extraño. La filosofía "natural" substituye a la "hablada". El discurso filosófico no es un saber con unos contenidos determinados, sino un ejercicio que afecta al hombre en su totalidad, transformándolo. La

3 "Vraye et naifve philosophie" (las traducciones son mías). 
filosofía, pues, no puede ser una profesión, una simple posesión de saberes, sino que, recuperando su sentido etimológico, se presenta como una actividad, y como una actividad vital. Sólo es posible hablar de discurso filosófico significativo en la medida que afecte a la vida, que, impregnándose en el sujeto, forme el juicio y las costumbres. No es extraño, pues, que Montaigne recomiende la filosofía para todas las edades y, en especial, en la infancia, y la convierta en el elemento central de sus propuestas sobre educación. Esto no es nuevo, pero en Montaigne la filosofía cobra un papel extraordinariamente vital, en tanto que debe llegar a naturalizarse, como sucede con Sócrates, el ejemplo más manejado por Montaigne, en quien la filosofía ha dejado de ser mero discurso, sus preceptos se han impregnado en él hasta tal punto que han dejado de ser tales, pasando a formar parte de su naturaleza.

Pero Montaigne no efectúa una simple vuelta atrás, recuperando la filosofía moral clásica. Produce un libro que no es un discurso cualquiera, sino que es consubstancial a su autor. Montaigne actúa como el espectador del estadio, pero es él mismo el observado. Hay un intento de conocimiento del yo, pero éste nunca puede ser definitivo ni verdadero. La crítica a la metafísica afecta también al estudio del yo. Entendiendo la metafísica como el intento de establecer una verdad intemporal, Montaigne la critica minando la base del edificio, la noción de substancia. Nada es estable, todo está en continuo movimiento, y el conocimiento, incluido el del yo, es imposible:

"No puedo fijar mi objeto. Va confuso y vacilante con una embriaguez natural. Lo tomo en ese punto, como está, en el instante en que me entretengo con él. No pinto el ser. Pinto el pasar: no el pasar de una edad a otra, o, como dice el pueblo, de siete en siete años, sino de día a día, de minuto a minuto. Es preciso adaptar mi historia al momento. Podré cambiar enseguida, no sólo de fortuna, sino también de intención. Es un registro de diversos y mutables accidentes y de imaginaciones no resueltas y, si sucede, contrarias; sea porque yo mismo soy otro, sea porque considere los temas por otras circunstancias y consideraciones. El caso es que acaso me contradiga, pero la verdad, como dijo Demades, no la contradigo en absoluto. Si mi alma pudiera asentarse, no me 
ensayaría, me resolvería; ella está siempre en aprendizaje y a prueba". (III, $2,782 b)^{4}$

Yo me escapo de mí mismo, me evaporo, no puedo asirme. Es necesario pues que la filosofía, alejada en palabras que pretenden encontrar una verdad externa e inalcanzable, retorne al sujeto, se convierta en un ejercicio para la formación del yo. El libro habla de muchas cosas, pero por encima de todo hay un único tema: Michel de Montaigne. Quien conoce el libro conoce su autor, pero no un relato autobiográfico al uso, ni una simple descripción física, sino que conoce los pensamientos del autor, el discurso que lo refleja, que lo encarna. Cuando leemos un capítulo de los Ensayos encontramos elementos que aparecen en otros desde otra perspectiva, a veces con contradicciones. Los temas se repiten, y todos giran en torno al yo que se ejercita, que se pone a prueba en su autoanálisis, que se construye en ese proceso reflexivo. Montaigne pretende llevar a cabo lo que para él es la única actividad filosófica posible: la producción de un libro como los Ensayos, esto es, un libro cuyo discurso corporalice a su autor, un libro escrito desde el yo y sobre el yo. Se entiende pues que los Ensayos sean un libro abierto, asistemático, heterogéneo, polisémico, con continuos añadidos. La filosofía de Montaigne, pues, no consiste sólo en las opiniones de Montaigne, sino sobre todo en la propia actividad de escritura del libro,

4 "Je ne puis asseurer mon object. Il va trouble et chancelant, d'une yvresse naturelle. Je le prens en ce point, comme il est, en l'instant que je m'amuse à luy. Je ne peints pas l'estre. Je peints le passage: non un passage d'aage en autre, ou, comme dict le peuple, de sept en sept ans, mais de jour en jour, de minute en minute. Il faut accommoder mon histoire à l'heure. Je pourray tantost changer, non de fortune seulement, mais aussi d'intention. C'est un contrerolle de divers et muables accidens et d'imaginations irresoluës et, quand il eschet, contraires ; soit que je sois autre moymesme, soit que je saissise les subjectspar autres circonstances et considerations. Tant y a que je me contradis bien à l'adventure, mais la vérité, comme disoit Demades, je ne la contredy point. Si mon ame pouvoit prendre pied, je m'essaierois pas, je me resoudrois; elle est toujours en apprentissage et en espreuve". Las dos primeras páginas del capítulo "Du repentir" son en cierto modo una declaración de principios de la actividad montaigneana. Un comentario excelente de estas páginas puede encontrarse en Erich Auerbach, Mimesis. Cap. XII. Trad. castellana del original alemán (Berna, 1946) en FCE, Madrid, 1983. 
esto es, no consiste solamente en lo que dice, sino también, y sobre todo, en lo que hace.

Montaigne, pues, filósofo. ¿Montaigne también filósofo moderno? La manera de introducir el sujeto en la actividad filosófica sitúa a Montaigne como un momento decisivo en la formación de la idea moderna de método, y por ende, de la nueva concepción de la historia, de la ciencia y del hombre 5 . Con Montaigne se toma conciencia de que es el sujeto el que establece el método, el yo que construye el discurso, y la filosofía cartesiana se formará a partir de esa idea. Montaigne se apercibe que la escritura del mundo y de su historia es un ejercicio teórico que revela una interpretación no cósmica, metafísica o religiosa, sino individual y subjetiva. Pero la tarea que se ha propuesto, la pintura de sí, no es por ello menos rigurosa: la descripción de sí mismo supone una atención constante, un sujetarse a la experiencia, que no está muy lejos del método experimental que se forja en el siglo XVII. Por otra parte, y precisamente por ese carácter individual y subjetivo, el discurso montaigneano diluye las categorías aristotélicas e introduce la idea de mezcla: "No probamos nada puro", nada se nos presenta aislada y nítidamente (II,20,655a) ${ }^{6}$. De este modo, reivindica el cuerpo (al menos tanto como el alma) como elemento de la condición humana $^{7}$, muestra la multiplicidad de la razón, la complejidad de los sentimientos, y la imposibilidad de una separación clara entre una y otra instancia. Esta "categoría" de mezcla, que se incorpora tanto al análisis del hombre mismo como al que éste hace respecto del mundo será desechada por Descartes que, aunque incorpora el discurso del yo como punto de partida para cualquier discurso filosófico, abandona la mezcla por cuanto que dificulta la superación del discurso individual, e incorpora al yo el método de la matemática.

${ }^{5}$ Desan ha trazado muy bien esta línea, que parte de Maquiavelo, sigue con La Ramée, Bodin y Montaigne, y culmina en Descartes. Véase Philippe Desan, Naissance de la métbode, Nizet, Paris, 1987.

6 "Nous ne goustons rien de pur".

${ }^{7}$ Se puede ver la filosofía de Montaigne como una filosofía del cuerpo, como lo hace Mathieu-Castellani, Montaigne, l'écriture de l'essai, Presses Universitaires de France, Paris, 1988. 
No es extraño entonces que Toulmin considere a Montaigne como un representante de una primera modernidad, más plural y escéptica, sustituida más tarde por una segunda modernidad, la cartesiana, más homogeneizante y formal ${ }^{8}$. El humanismo dejaría paso al racionalismo, el análisis de la condición humana a la búsqueda de la esencia humana. Toulmin acierta en que con el racionalismo, lo escrito, la lógica, lo universal y lo intemporal substituyen a lo oral, la retórica, lo particular y lo temporal que primaba en los discursos del siglo XVI. Toulmin añade, y también posiblemente con razón, que esa primera modernidad es más útil para abordar los problemas de hoy en día, frente al discurso de la segunda modernidad, que parece agotado. Pero si esto es así, en cualquier caso parece irrelevante hablar de primera modernidad o de premodernidad. De modo inverso puede llegarse a la misma conclusión: Montaigne no sería moderno por cuanto que no cree en la Razón como medio para situarse por encima de la tradición y hacer el mundo mejor, y prefiere el juicio, que es siempre particular y que da pie al diálogo, a confrontar opiniones diversas y hasta opuestas. Por tanto, no es cuestión de etiquetas, y aunque nos pusiésemos de acuerdo en los parámetros que definen la filosofia moderna, la complejidad de Montaigne es tal que impide ser clasificado con facilidad'

${ }^{8}$ Toulmin Stephen, Cosmopolis. The bidden agenda of modernity, Free Press, New York, 1990.

${ }^{9} \mathrm{El}$ escepticismo montaigneano, sostenible aún pese a Descartes o Kant, entronca con parte de la más importante epistemología contemporánea (pensemos en Kuhn, Feyerabend o Rorty). Pero la imposibilidad de acceder a la verdad, que hace que todo sea posible, no significa que todo sea igualmente asumible. Ahí reside la diferencia de Montaigne respecto de la posmodernidad: en ésta el relativismo supone juego, máscara, cuando en Montaigne aparece un deseo de sinceridad, de autenticidad. La sinceridad es un valor moral para Montaigne, y sin ella el libro consubstancial a su autor no es posible. En los Ensayos abundan las confesiones de sinceridad (aunque eso no significa que todo pueda ser dicho) y las críticas a la mentira. Ésta, aunque en ocasiones pueda ser útil, es siempre deshonesta, por cuanto que impide la comunicación: "Como nuestra inteligencia se conduce por la única vía de la palabra, el que la falsa traiciona la sociedad pública" (II,18,650a) ("Nostre intelligence se conduisant par la seule voye de la parolle, celuy qui la fauce, trahit la societé publique"). La sinceridad es condición para la vida social, y para la 
Aceptemos la singularidad de Montaigne, y hagámonos otra pregunta: no ya si Montaigne es un filósofo moderno, sino qué tiene Montaigne de actual. ¿Tiene sentido una filosofía como la que propone, consistente en ensayarse y escribir sobre sí mismo después de la crisis del sujeto y de la filosofía de la conciencia? ¿No nos está invitando Montaigne a escribir ficción más que a filosofar? Intentaré responder a esto señalando tres temas respecto de los que volver la mirada a Montaigne puede ser útil. Ya nos hemos referido a un aspecto de la actualidad de Montaigne: su posición particularista que mueve al diálogo y al acuerdo. En efecto, en un mundo multicultural como el que ahora vivimos, mundo que necesita acuerdos y consensos quizás esta actitud montaigneana de defender el placer de la discusión sea más adecuada ${ }^{10}$. En este sentido, puede ser útil para reflexionar sobre el problema de la universalidad y la particularidad. Recordemos unas cuantas frases representativas de los Ensayos. Nos advierte en el prefacio al lector que "Soy yo mismo la materia de mi libro" (Au lecteur, 9) ${ }^{11}$. Hablando de los historiadores, dice que representan "el hombre en general, del cual busco el conocimiento" (II,10,396c) ${ }^{12}$. Y en "Du repentir" escribe una de las frases más citadas: "cada hombre lleva la forma entera de la condición humana" (III,2,782b) ${ }^{13}$. Intentemos, aunque suponga romper el estilo montaigneano, transformar todo esto en un razonamiento: 1 . me estudio a mí mismo; 2. estudio al hombre; 3 . por tanto, estudiándome a mí mismo estudio al hombre. La filosofía y la ciencia modernas proceden de modo casuístico: estudian un número elevado de hombres para extraer cualidades comunes y determinar lo que es el hombre. Montaigne procede de diferente manera: parte de sí mismo para investigar la condición humana y el resto de hombres. Como bien señala Taylor, en el caso del yo el lenguaje es parte del objeto estudiado, y comoquiera que un lenguaje sólo existe en una comunidad lingǘstica, el yo nunca se describe

formación del sujeto. Sin sinceridad no hay libro ni actividad filosófica. Ésta no produce un discurso verdadero, pero sí honesto.

${ }^{10}$ Véase el capítulo "De l'art de conferer" (III,8, 899-922)

11 "Je suis moy-mesmes la matiere de mon livre"

12 "L'homme en general, de qui je cherche la cognoissance"

13 "Chaque homme porte la forme entiere de l'humaine condition" 
sin referencia a su entorno ${ }^{14}$. El estudio del yo se produce a partir de la diferencia, pero si ésta es percibida es en base a una condición común. Condición no es igual a esencia: no hay identidad, pero sí hay unos límites, que nos distinguen de los dioses y de las bestias. Las reflexiones que aparecen en los Ensayos respecto de los pueblos de América nos pueden servir para entender la posición montaigneana ${ }^{15}$. La idea predominante en la mayoría de los escritos del siglo XVI sobre los indígenas americanos es la siguiente: si formaban parte de la humanidad debían estar sometidos a las mismas leyes naturales que los europeos, esto es, significaba que tenían el mismo origen y formaban parte de la misma historia -la cristiana. Este planteamiento dejará paso en el siglo XVII a otro de más amplio alcance, basado en Descartes. La modernidad cartesiana supone una razón única por encima de las costumbres, y da lugar al proyecto ilustrado consistente en aplicar esa razón a todos los ámbitos de la vida, sacando a los hombres de la minoría de edad. La Ilustración presupone el universal Hombre, al cual los diversos hombres se han de adecuar. Sin embargo, Montaigne representa, en el siglo XVI, un punto de vista diferente, que mantiene la humanidad de los indígenas americanos al tiempo que defiende un origen y una historia diferentes de la europea. Montaigne mina la idea de ley natural: si existiese todos los hombres la llevarían impresa en su conciencia y no se daría la diversidad que observamos; y comoquiera que la diversidad es un hecho, no tiene sentido hablar de desviaciones o de falta de progreso, sino más bien de diferencias ${ }^{16}$. La razón, para Montaigne, tiene muchas caras, y lejos de unificar la conducta, la diversifica. La paradoja es que la razón nos distingue del resto de especies, nos hace humanos, pero es a través de ella que nos diferenciamos entre nosotros. Por eso no es procedente hablar del Hombre, sino de los hombres, con toda su pluralidad de historias y su diversidad cultural.

14 Taylor, Charles, Sources of the Self. The Making of the Modern Identity, Harvard University Press, Cambridge, 1989. Hay traducción castellana a cargo de Ana Lizón en Paidós, Barcelona-Buenos Aires, 1996.

${ }^{15}$ Lo que sigue está desarrollado en J.L. Llinàs, "Una crítica avant la lettre a la raó moderna: Montaigne i el problema de la interculturalitat", en Taula, quaderns de pensament (Palma de Mallorca), $\mathrm{n}^{\circ}$ 33-34, 2000, pp. 179-185.

16 Véase "Apologie de Raimond Sebond", II,12, en especial la primera parte, donde se responde a la primera objeción efectuada a Sebond. 
El mundo multicultural actual se mueve entre dos peligros: la jerarquización de culturas en base a unos criterios etnocéntricos y la aceptación de la inconmensurabilidad de culturas que puede justificar el exterminio de algunas de ellas. Tal vez una relectura de Montaigne sea útil para combinar la aceptación conjunta de la multiculturalidad y de la comunidad humana. Los hombres son diferentes, pero no inconmensurables, es decir, la comunicación es posible (por eso Montaigne recomienda la historia y la etnología para el estudio de sî). Cada uno de ellos particulariza una humanidad que se caracteriza precisamente por la pluralidad de su condición. En otras palabras, parece ser posible conjugar el relativismo cultural con el principio de la igual dignidad de las culturas. ¿Cómo resolver, desde esta posición, los conflictos culturales? En primer lugar, Montaigne prefiere la costumbre a la razón. Si la costumbre cumple con su función y la razón es múltiple, no tiene sentido cambiar por cambiar. Montaigne rechaza con contundencia lo que denomina nouvelletez. La costumbre no tiene más fundamento que el tiempo que valida su utilidad. Aplicado, por ejemplo, a la religión, esto significa que delante de la imposibilidad de verificar cuál es la verdadera religión no es conveniente cambiar si ello ha de suponer guerra y destrucción. En segundo lugar, la conciencia del etnocentrismo no imposibilita el juicio, más bien para Montaigne es al contrario: estamos en condiciones de juzgar mejor puesto que somos conscientes que nos situamos en una perspectiva determinada, no en la absoluta o "buena". Por ejemplo, Montaigne puede permitirse defender que los "caníbales" no son más bárbaros que los europeos y al mismo tiempo criticar su canibalismo. En tercer lugar, si no existe ley natural hay que manejarse con la ley positiva. Esta no es universal, sino cambiante, contingente y a veces arbitraria, pero la alternativa es el desorden y la imposibilidad de la vida colectiva. Esto no significa que haya que seguir las normas de manera acrítica; pueden ser criticadas, precisamente porque son "municipales", pero teniendo en cuenta que hay que evitar el cambio por el cambio. Dicho de otra manera, si bien Montaigne puede ser visto como un conservador en la medida que defiende el orden proporcionado por la costumbre, también su planteamiento permite el cambio social: frente a la Razón intemporal, única, buena, que nos dirige hacia un solo camino, darse cuenta de la convencionalidad del orden nos permite cambiarlo si así lo consideramos y al tiempo nos hace más responsables de nuestras decisiones. En cuarto lugar, la propuesta de Montaigne para evitar el etnocentrismo consiste en viajar -real o virtualmente. El contacto con los 
demás nos muestra la diversidad de la condición humana, a la vez que constatamos que la comunicación es posible. Montaigne afirma que cada hombre lleva en él la forma entera de la condición humana (III,2, 805b), lo que significa que, pese a las diferencias, todos somos hombres. Nada humano puede sernos ajeno, todo aquello extraño de otra cultura es potencialmente nuestra forma concreta. Por eso Montaigne propone educar en la flexibilidad, en poder hacer de todo, puesto que así no quedaremos encorsetados en una sola forma de vida, en un único punto de vista.

Esta posición puede parecer ingenua, ya que empieza a ser problemática cuando las diferencias afectan a cuestiones consideradas esenciales. Por ejemplo, nosotros, que hemos sido educados en una cultura que lo considera aberrante, difícilmente nos adaptaremos al infanticidio. Esto significa, para Montaigne, que no todo es igualmente digerible. Pero la causa del rechazo no radica en la Razón o en la Ley Natural, sino en que contradice los fundamentos en los que descansa nuestra sociedad. Quizás Montaigne no dé solución a los problemas derivados de la multiculturalidad, pero apunta un camino, que no pasa por las ideas derivadas de una posición monogénica (Razón, Progreso, Hombre...) sino por constatar que un origen cultural diverso no supone la pérdida de una misma condición humana y que precisamente por ello el diálogo siempre es posible. Dicho de otra manera, con Montaigne la conciencia de la debilidad de la razón nos impele a repensar los problemas desde las diferencias, desde los hombres.

Otro retorno a Montaigne aparece en el curso de la reflexión sobre la identidad personal. Si el tema de los Ensayos es el propio Montaigne, la pregunta que sostiene el libro es la pregunta por el yo. Y la manera de plantear esa pregunta y emprender la respuesta muestra la peculiaridad de la pregunta. Estudiar el yo no es lo mismo que estudiar un objeto cualquiera, dado que cuando el yo es el objeto de estudio, no podemos considerarlo absolutamente, ni independientemente de las interpretaciones que de él ofrece un sujeto cualquiera, ni efectuar sobre él una descripción explícita y sin referencia a su entorno. La pregunta por el yo parece requerir tener en cuenta la manera en que las cosas son significativas para mí y la consideración que tengo sobre mí mismo. Los Ensayos no sólo ponen esto de manifiesto, sino también una doble necesidad: la de que el estudio del yo incluya sus autointerpretaciones; y la necesidad de efectuar una descripción inacabada, puesto que toda descripción del yo debe incluir la del lenguaje en que se produce, y éste sólo existe socialmente, lo que impide que la 
descripción pueda concluir ${ }^{17}$. En otros términos, lo que muestran los Ensayos es que el yo no es una cosa, y por tanto no se puede hacer ciencia sobre él como se lleva a cabo la ciencia sobre las cosas. Estudiar el yo supone plantear la cuestión de la identidad personal, esto es, no sólo la identidad que viene dada por la ubicación espacio-temporal de un objeto, sino la que aparece al determinar su singularidad y al encontrar algo permanente al cambio. $\mathrm{Y}$, cuando el objeto estudiado es el yo, la objetivación, que es posible si el objeto de estudio es el hombre (podemos responder en términos biológicos -el genoma y el sistema inmunológico), se resiste. La pregunta remite a una identidad subjetiva, reflexiva, que no se puede reducir a un nombre o a un objeto.

La actualidad de Montaigne en este tema aparece en tanto que representa una tercera vía entre el cartesianismo y sus críticos, esto es, entre situar al yo como verdad primigenia en base al cual se produce el método de adquisición y la construcción del edificio de verdades, y ver al cogito como algo extraño y complejo, objeto de interpretación, pura ilusión, del cual lo mejor que podemos hacer es olvidarnos. Cierto que Montaigne anticipa a Descartes al situar al yo en el centro de la reflexión sobre el conocimiento, pero no pretende construir un método para investigar la verdad en las ciencias. Cierto también que Montaigne presenta afinidades con los críticos de la modernidad, empezando por Nietzsche y siguiendo con autores como Derrida o Foucault, pero no es posmoderno en el sentido que proponga la disolución del sujeto. Más bien lo que se propone es una aproximación peculiar al yo, que enlaza con el clásico precepto délfico "conócete a ti mismo" y a la vez con posiciones contemporáneas como la de Paul Ricoeur ${ }^{18}$. La buena vida es la vida examinada, pero este examen muestra la imposibilidad que el sujeto se fundamente a sí mismo, sujeto que no puede asirse, sostenerse, sujeto que está siempre evaporándose. La identidad personal, entonces, sólo aparece como identidad narrativa, como historia de una vida. Es a través del relato que aparece el "quien", y es a través de ese mismo relato que el sujeto se comprende a sí mismo como inacabable y

${ }^{17}$ Véase Taylor, op. cit., cap. 2.

${ }^{18}$ Dejaré de lado las diferencias entre ambos autores para señalar tan sólo puntos de contacto. 
abierto siempre a la novedad ${ }^{19}$. Entre la apología del cogito y su eliminación, el yo se reconoce a sí mismo en su relación con el otro y en la mediación de los signos y los textos ${ }^{20}$. En definitiva, Montaigne supone una aproximación contemporánea a la cuestión de la identidad, en la que el yo es no tanto fundamento $\mathrm{u}$ origen como tarea, formado por gran número de elementos que le proporcionan complejidad e inestabilidad, y de ahí la necesidad de traducción, esto es, de narrar la vida ${ }^{21}$.

El tercer tema en el que un retorno a Montaigne es aconsejable tiene que ver con el anterior, en la medida que, tal como plantea la cuestión de la identidad personal, ésta implica una determinada visión de la tarea filosófica y una propuesta de un discurso filosófico alternativo al discurso objetivo y verdadero. Ya hemos hablado de cómo concibe la actividad filosófica, su propia actividad. Busca cómo estar en un mundo en que el acceso al ser es imposible (II,12,586a) y tan solo queda ponerse a prueba, ensayarse. Y el ensayo, en Montaigne, se produce en la escritura, no en una escritura automática registradora continua de hechos, sino en una escritura reflexiva, que muestra y al tiempo somete a prueba el pensamiento. Escribiendo este libro único, consubstancial a su autor, el pensamiento se transforma en acción, una acción que a su vez es nueva materia de reflexión. En este proceso sin fin Montaigne hace su libro al tiempo que el libro le hace a él. Hacer filosofía es, para Montaigne, escribir, aunque tiene presente los problemas que había planteado Platón en la Carta VII. Lo escrito simboliza lo muerto, sólo transmite ideas, conceptos, descontextualiza, separa. Sólo se puede legitimar si refleja la vida, si es discurso del yo. Hacer filosofía, pues,

19 Recordemos el final de Mémoire, Histoire, Oubli, Seuil, Paris, 2000, p. 656: «Bajo la historia, la memoria y el olvido. Bajo la memoria y el olvido, la vida. Pero escribir la vida es otra historia. Lo inacabable" ("Sous l'histoire, la mémoire et l'oubli. Sous la mémoire et l'oubli, la vie. Mais écrire la vie est une autre histoire. Inachèvement") Y también el principio de "De la vanité": «Quién no ve que he tomado un camino por el cual, sin reposo y sin esfuerzo, iré mientras haya tinta y papel en el mundo? " (Qui ne voit que j'ay pris une route par laquelle, sans cesse et sans travail, j'iray autant qu'il y aura d'ancre et de papier au monde?') (III,9,922b).

${ }^{20}$ Véase Essais II,18,648c, y Paul Ricoeur, Du texte a l'action, Seuil, Paris, 1986.

${ }^{21}$ Esto es lo que hace Montaigne y esto es también, salvando las obvias diferencias, que no son objeto de este escrito, lo que se propone Ricoeur. Véase P. Ricoeur, «Le paradigme de la traductions, en Esprit, 1999, pp. 8-19. 
es ensayarse, y el ensayo deviene la forma filosófica por excelencia. En este sentido, el siglo XX ha supuesto una profunda reflexión sobre lo que es el género filosófico. Adorno, por ejemplo, ha reflexionado sobre esta forma filosófica que se presenta como alternativa a las reglas del método de Descartes, esto es, al ideal de percepción clara y distinta, a la idea de partir de lo más simple, y a la idea que es posible resolver del todo el tema abordado 22 . Aunque quizás sería más correcto afirmar que es Descartes quien propone un método contra esa filosofía siempre expuesta al error que es el ensayo. Una vez producido el triunfo de la opción cartesiana, que configura la modernidad, y su posterior entrada en crisis, de lo que se trata es de reflexionar sobre la vigencia de la posibilidad del ensayo, del pensamiento discontinuo. $\mathrm{Y}$ cuando Adorno señala las características del ensayo al lector se le aparecen los Ensayos de Montaigne: no busca reducirlo todo a un principio; se alza contra la doctrina de que lo cambiante es indigno de la filosofía; defiende lo fáctico frente al concepto puro; se opone a la idea de que verdad e historia se contraponen; suspende el concepto tradicional de método; no busca definir conceptos sino que los usa tal y como los concibe; y, finalmente, se produce básicamente como crítica. Montaigne presenta pues una alternativa a la actividad filosófica, una alternativa que supone dar más importancia a la forma que al contenido y que al tiempo singulariza la filosofía ${ }^{23}$. Escribiendo sobre sí mismo crea sin que por ello esté haciendo arte. Pero si no hace ni filosofía en sentido académico ni arte, ¿qué hace? ¿No es, como señalábamos más arriba, pura ficción? La peculiaridad y la paradoja de los Ensayos reside precisamente en esto, que es ficción al tiempo que realidad, que es reflexión teórica a la vez que concreción práctica. La suya es una filosofía toda acción, aplicación de unas ideas que aparecen mezcladas en el discurso práctico. En los Ensayos teoría y praxis no se separan, y la filosofía y su aplicación son uno. $\mathrm{Y}$ sin embargo, el libro supone una dislocación: pensándose Montaigne se hace, y, en la medida que se sitúa en un espacio distinto del hombre que lo escribe, espacio cerrado e inmóvil, el libro substancializa a su autor. La paradoja

${ }^{22}$ Adorno, T. W., "El ensayo como forma", en Notas de literatura, Ariel, Barcelona, 1962, pp. 11-36. Trad. de Manuel Sacristán de Noten zur Literatur, Suhrkamp Verlag, Frankfurt am Main, 1962.

${ }^{23}$ Como sucedía antes con Ricoeur, dejo de lado las diferencias con Adorno para otra ocasión, y me limito a señalar la conexión existente. 
reside en un libro que nunca puede darse por terminado, en la medida que su autor quiere ser en otro sitio distinto del papel escrito. Los Ensayos son la tumba de Montaigne, y el espejo de su práctica filosófica. Creación, filosofía y escritura no son separables. La actividad filosófica da lugar a la formación de sí, a la creación del yo, y produce para ello un tipo de escritura que, para Montaigne, es la única filosóficamente relevante: la auto-bio-grafía. Tal vez una lectura de los Ensayos en esta línea contribuya al esclarecimiento de qué significa hacer filosofía.

\title{
Resumen
}

El artículo se divide en tres partes. En primer lugar, se comenta la crítica de Montaigne a una determinada filosofía y se defiende la consideración de Montaigne como filósofo. En segundo lugar, se intenta situar a Montaigne respecto de la filosofía moderna. En tercer lugar, más allá de su modernidad, se comenta su utilidad para reflexionar algunos problemas contemporáneos, como el de la multiculturalidad, el de la identidad personal y el de la justificación de la actividad filosófica.

Palabras clave: "Montaigne", "filosofía moderna", "multiculturalidad", "ensayo", "escritura filosófica", "identidad personal"

\begin{abstract}
This article is divided into three parts. Firstly, there is a discussion on Montaigne's critique of a given philosophy, and a defense of the consideration of Montaigne as a philosopher. Secondly, a situation of Montaigne regarding modern philosophy is attempted. Thirdly, beyond his modernity, his usefulness for reflecting about some contemporary problems, such as that of multiculturalism, that of personal identity and that of philosophical activity, is discussed.
\end{abstract}

Key words. "Montaigne", "modern philosophy", "multiculturalism", “essay", "philosophical writing", "personal identity" 